\title{
Effect of Ligation Method on Maxillary Arch Force/Moment Systems for a Simulated Lingual Incisor Malalignment
}

\author{
Surbhi Seru ${ }^{\mathrm{a}}$, Dan L Romanyk ${ }^{\mathrm{b}}$, Roger W Toogood ${ }^{\mathrm{c}}$, Jason P Carey ${ }^{\mathrm{d}}$ and Paul W. Major ${ }^{\mathrm{e}, *}$ \\ ${ }^{a}$ Lab Coordinator, Orthodontic Biomechanics Laboratory, University of Alberta, Edmonton, $A B$ \\ ${ }^{b}$ Postdoctoral Fellow, School of Dentistry, University of Alberta, Edmonton, AB \\ ${ }^{c}$ Associate Professor, Department of Mechanical Engineering, University of Alberta, Edmonton, AB \\ ${ }^{d}$ Professor, Associate Chair Undergraduate Program (MecE), Department of Mechanical Engineering, University of \\ Alberta, Edmonton, $A B$ \\ ${ }^{e}$ Professor, Senior Associate Dean, Faculty of Medicine and Dentistry, University of Alberta, Edmonton, AB
}

\begin{abstract}
Introduction: The objectives of this study were to determine whether there is a difference in the magnitude of forces and moments produced by elastic ligation when compared to passive ligation, and whether these forces and moments propagate differently along the arch for the two ligation types. A lingual incisor malalignment was used in this study.

Methods: The Orthodontic Simulator (OSIM) was used to quantify the three-dimensional forces and moments applied on the teeth given a lingually displaced incisor. A repeated measures MANOVA was performed to statistically analyze the data.

Results: The interaction factor illustrated convincing evidence that there is a difference in maximum force and moment values for all outcome variables between ligation types considering all tooth positions along the arch. The mean differences for $F_{X}$ and $F_{Y}$ between ligation types were found to be clinically significant, with values for elastic ligation consistently higher than passive ligation.

Conclusion: It was found that the maximum forces and moments produced by elastic ligation are greater than those produced by passive ligation and that the magnitude of this difference for the mesiodistal and buccolingual forces is clinically relevant. Additionally, it was determined that elastic ligation causes forces and moments to propagate further along the arch than passive ligation for all outcome variables.
\end{abstract}

Keywords: Elastic ligation, force measurement, lingual incisor, orthodontics, orthodontic simulator, passive ligation.

\section{INTRODUCTION}

In orthodontics, ligation method refers to the means by which an archwire is held inside the bracket. Conventionally, elastic or metallic ties were used to hold the archwire in place; however more recently ligating methods have been designed and built directly into brackets. Currently the three most common ligation systems are active- and passive selfligation and conventional elastic ligation. Research has shown that different ligation types produce different force and moment systems along the orthodontic arch; specifically, passive self-ligation has been found to decrease the forces and moments produced when compared to elastic ligation, [1-5] which may lead to increased patient comfort [6]. This is because elastic ligation actively holds the wire in place

*Address correspondence to the author at the 5-478, Edmonton Clinic Health Academy (ECHA), University of Alberta, 11405-87 Ave NW, Edmonton, AB, Canada, T6G 1C9; Tel: 780-492-3312; Fax: 780-492-7536; E-mail: major@ualberta.ca against the bracket, whereas passive ligation simply guides the wire while leaving room for movement. A better understanding of the mechanics of passive and elastic ligation is desired in order to be able to improve overall orthodontic treatment [7].

The Orthodontic Simulator (OSIM) in Fig. (1) is an electromechanical system developed by researchers and clinicians at the University of Alberta to quantify the threedimensional forces and moments applied on the teeth given specific orthodontic conditions, described in detail by Badawi et al. [8-10].

The objectives of this study are to determine whether there is a difference in the magnitude of forces and moments produced by elastic ligation when compared to passive ligation during alignment of a lingually displaced maxillary lateral incisor, and whether these forces and moments propagate differently along the arch for the two ligation types. The hypotheses are that the magnitude of forces and 


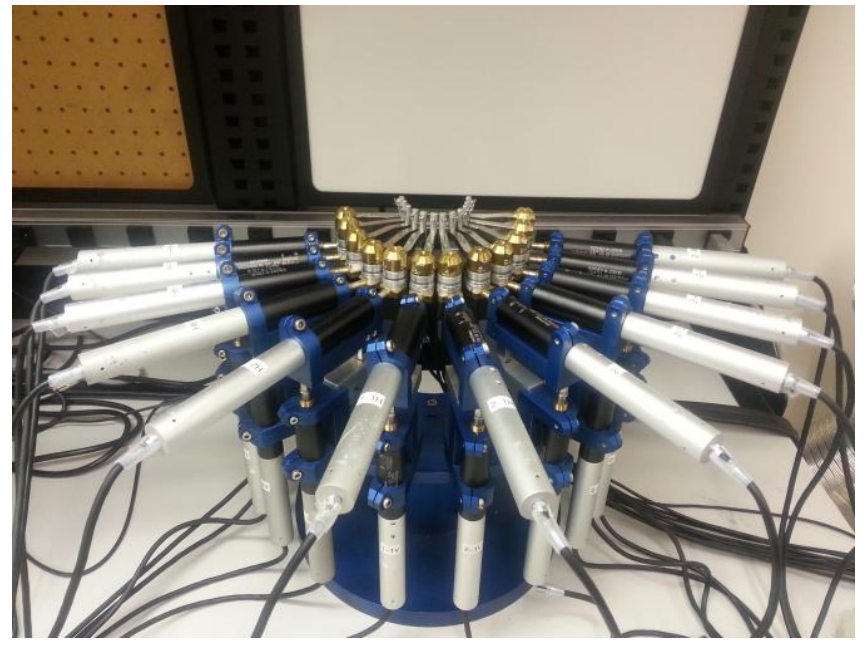

Fig. (1). Orthodontic Simulator in a neutral arch position.

moments will be greater for elastic ligation than passive ligation, and that elastic ligation will propagate forces further along the arch than passive ligation. These hypotheses are based on the results from previous studies analyzing a high canine malocclusion $[1,11]$.

\section{METHODS}

The OSIM was utilized to quantitatively analyze the forces and moments produced along the arch. The forces are measured in Newtons $(\mathrm{N})$ and the moments are measured in Newton-millimeters (Nmm). The OSIM has 12 teeth arranged in an arch form, with each tooth attached to a sixaxis load cell. Vertical and horizontal micrometers connected to each tooth enable the user to move the teeth in the buccolingual (Y) or occlusogingival (Z) directions. Movement is measured in millimeters $(\mathrm{mm})$.

Since the forces and moments are measured at the load cell, it is necessary to transform these values from the load cell to the bracket to determine what forces and moments are experienced at the bracket itself. This is accomplished by a series of matrix multiplications called Jacobian transformations, described in detail as they function with the OSIM by Fok et al. [11]. The one difference is that the current experiment analyzes forces and moments produced at the bracket rather than at the tooth's center of resistance.

Other than the difference in ligation type, the testing procedure was the same for every trial. The OSIM was placed in a temperature chamber and warmed to $37^{\circ}$ Celsius to mimic biological conditions. The teeth were then put into a neutral arch position where, if ligated passively, minimal forces $(<0.09 \mathrm{~N})$ and moments $(<0.4 \mathrm{Nmm})$ would be produced on the teeth. From the neutral position, tooth 1-2 was moved lingually $4 \mathrm{~mm}$; this was the starting position for the experiment. A wire was inserted in the brackets and secured using either passive or elastic ligation. From $4 \mathrm{~mm}$, tooth 1-2 was then returned to the neutral arch position in $0.2 \mathrm{~mm}$ increments, totaling 21 steps. Force and moment data were recorded for all 12 teeth.

A pilot study was conducted to determine the sample size required for this experiment and it was concluded that in order to be able to detect a force of $0.09 \mathrm{~N}$ and a moment of
$0.4 \mathrm{Nmm}$ with a 0.05 level of significance, a sample size of 45 trials per ligation type would be sufficient. In both the pilot study and the overall experiment, the trials were randomized between passive and elastic ligation.

The brackets mounted on the teeth were self-ligating Damon 3MX brackets (Ormco, Orange, CA, USA). These brackets were used for all the trials; however, the gates of the brackets were left in the open position in the elastic ligation trials. The wires used for this experiment were Damon series CuNiTi 0.014 inch round wires (Ormco, Orange, CA, USA) and the elastics used for the elastic ligation trials were Power O 0.120 inch grey elastics (Ormco, Orange, CA, USA). A Straight Shooter Ligature Gun (TP Orthodontics, La Porte, IN, USA) was used to ensure the elastics were stressed uniformly and consistently before application. Due to the size of the brackets on 1-6 and 2-6, it was not possible to elastically ligate these teeth using the same elastics. Thus, even in the elastic trials, they were ligated passively by closing the gate on the bracket rather than using an elastic ligature.

The mesiodistal force $\left(F_{X}\right)$ represents resistance to sliding, with positive $\mathrm{X}$ in the distal direction for quadrant 1 and mesial direction for quadrant 2 . The buccolingual force $\left(F_{Y}\right)$ reflects the wire flexing toward the front or back of the slot, and is expected to be the largest force in this experiment because it is in the direction of the movement. This also means that in a similar clinical setting, $F_{Y}$ will be the largest force felt by the patient. Positive $\mathrm{Y}$ is in the buccal direction. In this experiment, occlusogingival force $\left(\mathrm{F}_{\mathrm{Z}}\right)$ represents errors in bracket or wire positioning on the OSIM since there is no expected movement of the tooth or wire in the $\mathrm{Z}$ direction. Positive $\mathrm{X}$ is in the gingival direction.

$\mathrm{M}_{\mathrm{X}}$ values represent torque applied on the teeth moving the root of the tooth in the buccal or lingual direction. Since this experiment uses round wires, no engagement between the wire and the slot is expected, which means the $\mathrm{M}_{\mathrm{X}}$ values should be close to zero. $M_{Y}$ values represent the mesiodistal tipping moment on the teeth and $\mathrm{M}_{\mathrm{Z}}$ represents rotational moment of the tooth along its long axis.

SPSS 20.0 (IBM, Armonk, NY, USA) was used to conduct repeated measures MANOVA with a 0.05 level of significance. 1-6 and 2-6 were excluded from the statistical analysis since these teeth were passively ligated for all trials. The within-subjects factor was tooth position, with 10 levels for the remaining 10 teeth along the arch. The betweensubjects factor was ligation type, with two levels, passive and elastic. The outcome variables were $\mathrm{F}_{\mathrm{X}}, \mathrm{F}_{\mathrm{Y}}, \mathrm{F}_{\mathrm{Z}}, \mathrm{M}_{\mathrm{X}}, \mathrm{M}_{\mathrm{Y}}$, and $\mathrm{M}_{\mathrm{Z}}$. For each trial, per tooth, the force and moment values with the largest magnitude out of the 21 steps were used in the statistical analysis. For the pairwise comparisons, the absolute values were used in order to accurately determine the mean differences between the ligation types. This is acceptable because the signs of the values are arbitrary and simply indicate direction, whereas this study was more interested in the difference in magnitude of force and moment values between the ligation types.

It has been found that forces as low as $0.2 \mathrm{~N}$ have generated tooth movement [12], and moments in the range of 5-20 Nmm cause tooth rotation [13]. Although it is 
recognized that the evidence to support minimum force and moment thresholds is not conclusive, this study will use 0.2 $\mathrm{N}$ and $5 \mathrm{Nmm}$ as clinically relevant values when discussing forces and moments, respectively.

\section{RESULTS}

Although Levene's test of equality of variances was significant for some of the data, equal variances can be assumed since the sample size was large with an equal number of trials per ligation type, and because MANOVA is robust to discrepancies in variance. The results from Mauchly's test indicated that the assumption of sphericity had been violated. Therefore, degrees of freedom were corrected using Greenhouse-Geyser estimates of sphericity.

The results of the repeated measures MANOVA indicated that there was a statistically significant interaction effect between tooth position and ligation type for all six outcome variables $\left(\mathrm{F}_{\mathrm{X}}, \mathrm{F}_{\mathrm{Y}}, \mathrm{F}_{\mathrm{Z}}, \mathrm{M}_{\mathrm{X}}, \mathrm{M}_{\mathrm{Y}}\right.$, and $\left.\mathrm{M}_{\mathrm{Z}}\right)$ with $\mathrm{p}$ values $<0.001$ when all teeth positions were considered jointly. This implied that the propagation of forces and moments along the arch differed significantly between the ligation types for all the outcome variables, but did not indicate the nature of the difference.

There was also a statistically significant effect of ligation type, as shown in Table $\mathbf{1}$, for $\mathrm{F}_{\mathrm{X}}, \mathrm{F}_{\mathrm{Y}}, \mathrm{F}_{\mathrm{Z}}, \mathrm{M}_{\mathrm{Y}}$, and $\mathrm{M}_{\mathrm{Z}}$, with $\mathrm{p}$-values $<0.001$. The $\mathrm{p}$-value for $\mathrm{M}_{\mathrm{X}}$ was 0.319 , indicating no statistical significance. Values were consistently greater for elastic ligation than passive ligation, with the mean differences for $F_{X}$ and $F_{Y}$ greater than the previously mentioned clinically relevant threshold of $0.2 \mathrm{~N}$. The means and mean differences for each tooth for $F_{X}$ and $F_{Y}$ are reported in Tables 2 and $\mathbf{3}$. The mean differences for $F_{Z}, M_{X}$, $\mathrm{M}_{\mathrm{Y}}$, and $\mathrm{M}_{\mathrm{Z}}$ were below the clinically relevant thresholds of $0.2 \mathrm{~N}$ and $5 \mathrm{Nmm}$ for forces and moments, respectively.

The estimated marginal mean plots (Figs. 2-7) qualitatively illustrate the differences in force and moment values and propagation of forces and moments along the arch between the ligation types based on tooth position. Both

Table 1. Ligation type pairwise comparisons (for all teeth).

\begin{tabular}{|c|c|c|c|c|c|}
\hline Outcome variable & Mean difference $^{a}$ & Standard error & p-value & \multicolumn{2}{|c|}{$95 \%$ confidence interval } \\
\hline $\mathrm{F}_{\mathrm{X}}$ & $0.298^{b}$ & 0.006 & $<0.001$ & 0.287 & 0.310 \\
\hline $\mathrm{F}_{\mathrm{Y}}$ & $0.209^{\mathrm{b}}$ & 0.007 & $<0.001$ & 0.195 & 0.222 \\
\hline $\mathrm{F}_{\mathrm{Z}}$ & $0.020^{\mathrm{b}}$ & 0.002 & $<0.001$ & 0.017 & 0.024 \\
\hline $\mathrm{M}_{\mathrm{Y}}$ & $2.339^{\mathrm{b}}$ & 0.050 & $<0.001$ & 2.238 & 2.439 \\
\hline $\mathrm{M}_{\mathrm{Z}}$ & $1.744^{\mathrm{b}}$ & 0.042 & $<0.001$ & 1.661 & 1.828 \\
\hline
\end{tabular}

${ }^{a}$ The mean difference is reported as (Elastic) - (Passive).

${ }^{\mathrm{b}}$ The mean difference is significant at the 0.05 level.

Table 2. $F_{X}$ pairwise comparisons (per tooth).

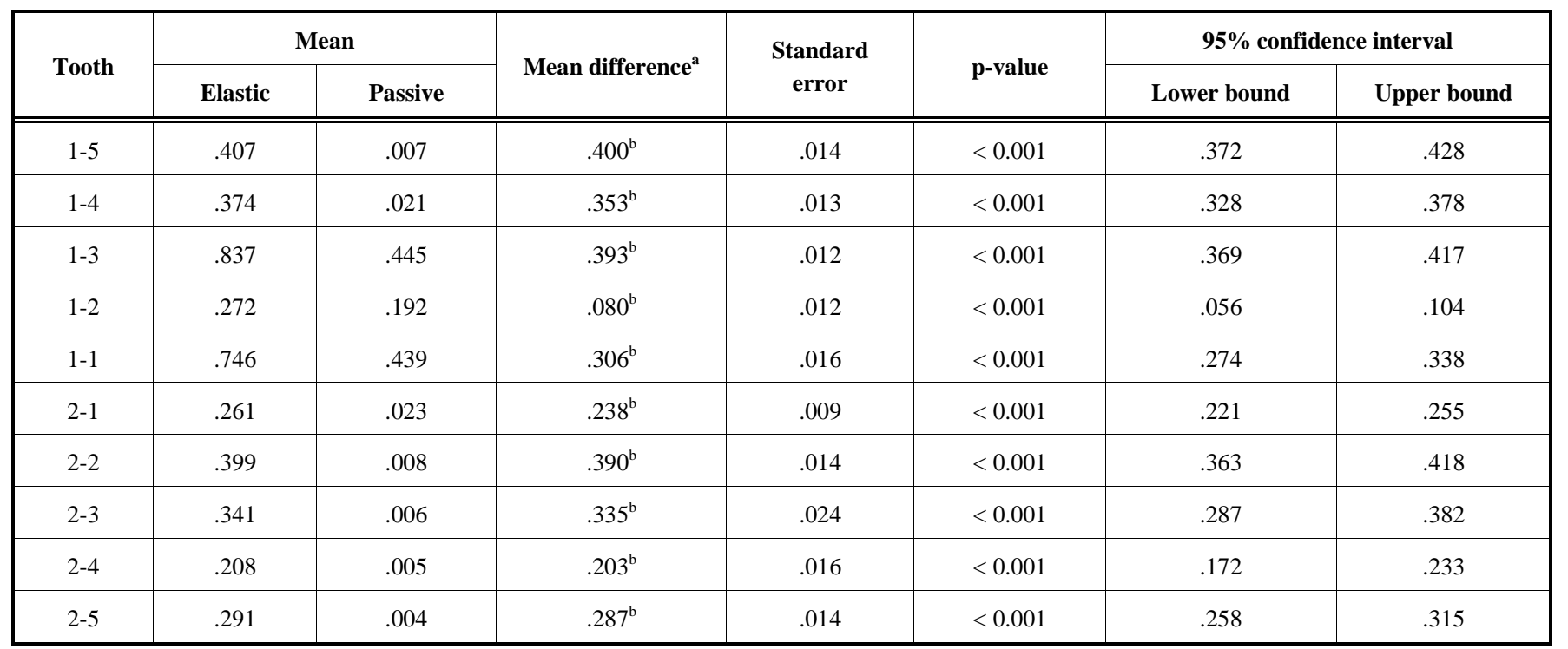

${ }^{a}$ The mean difference is reported as (Elastic) - (Passive).

${ }^{\mathrm{b}}$ The mean difference is significant at the 0.05 level. 
Table 3. F $_{\mathrm{Y}}$ pairwise comparisons (per tooth).

\begin{tabular}{|c|c|c|c|c|c|c|c|}
\hline Tooth & \multicolumn{2}{|c|}{ Mean } & Mean difference ${ }^{a}$ & $\begin{array}{c}\text { Standard } \\
\text { error }\end{array}$ & p-value & \multicolumn{2}{|c|}{$95 \%$ confidence interval } \\
\hline $1-5$ & .349 & .076 & $.273^{\mathrm{b}}$ & .013 & $<0.001$ & .248 & .298 \\
\hline $1-4$ & .209 & .197 & .012 & .017 & 0.474 & -.022 & .047 \\
\hline $1-3$ & .670 & .739 & $-.069^{\mathrm{b}}$ & .018 & $<0.001$ & -.106 & -.033 \\
\hline $1-1$ & .857 & 1.036 & $-.179^{\mathrm{b}}$ & .018 & $<0.001$ & -.214 & -.144 \\
\hline $2-1$ & .794 & .298 & $.496^{\mathrm{b}}$ & .013 & $<0.001$ & .471 & .521 \\
\hline $2-2$ & .471 & .020 & $.452^{\mathrm{b}}$ & .010 & $<0.001$ & .432 & .471 \\
\hline $2-3$ & .379 & .009 & $.370^{\mathrm{b}}$ & .012 & $<0.001$ & .347 & .393 \\
\hline
\end{tabular}

${ }^{a}$ The mean difference is reported as (Elastic) - (Passive).

${ }^{\mathrm{b}}$ The mean difference is significant at the 0.05 level.

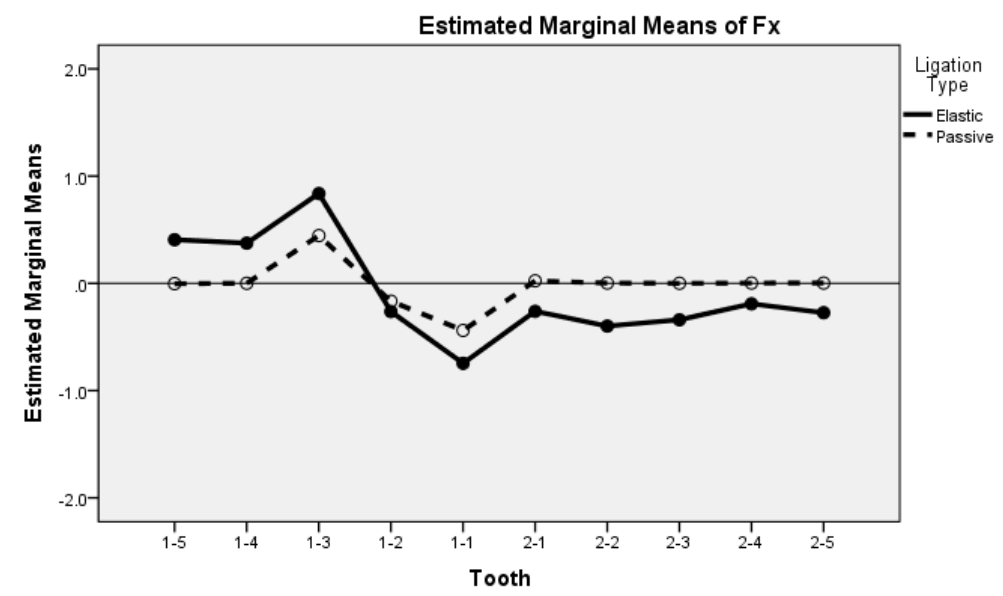

Fig. (2). Estimated marginal means of $F_{X}$.

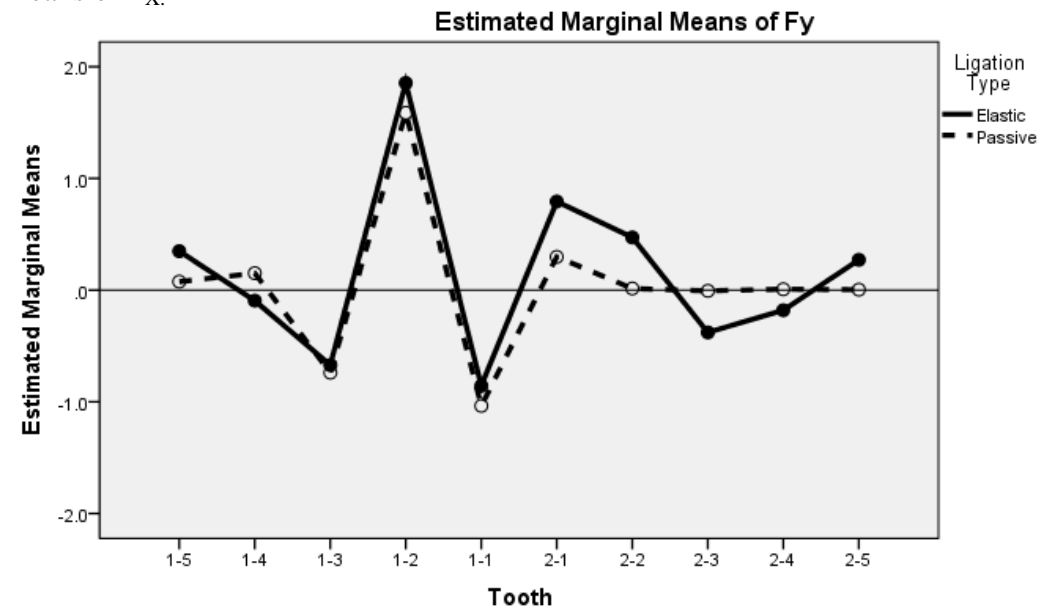

Fig. (3). Estimated marginal means of $\mathrm{F}_{\mathrm{Y}}$.

elastic and passive ligation exhibit similar trends for the tooth that was moved (1-2) and the two teeth on either side (1-3 and 1-1) for all outcome variables. Beyond that, however, the marginal means for elastic ligation seem to propagate further along the arch than passive ligation. It is also apparent that the estimated marginal means for the elastic ligation trials are generally higher (in absolute terms) than the passive ligation trials. 


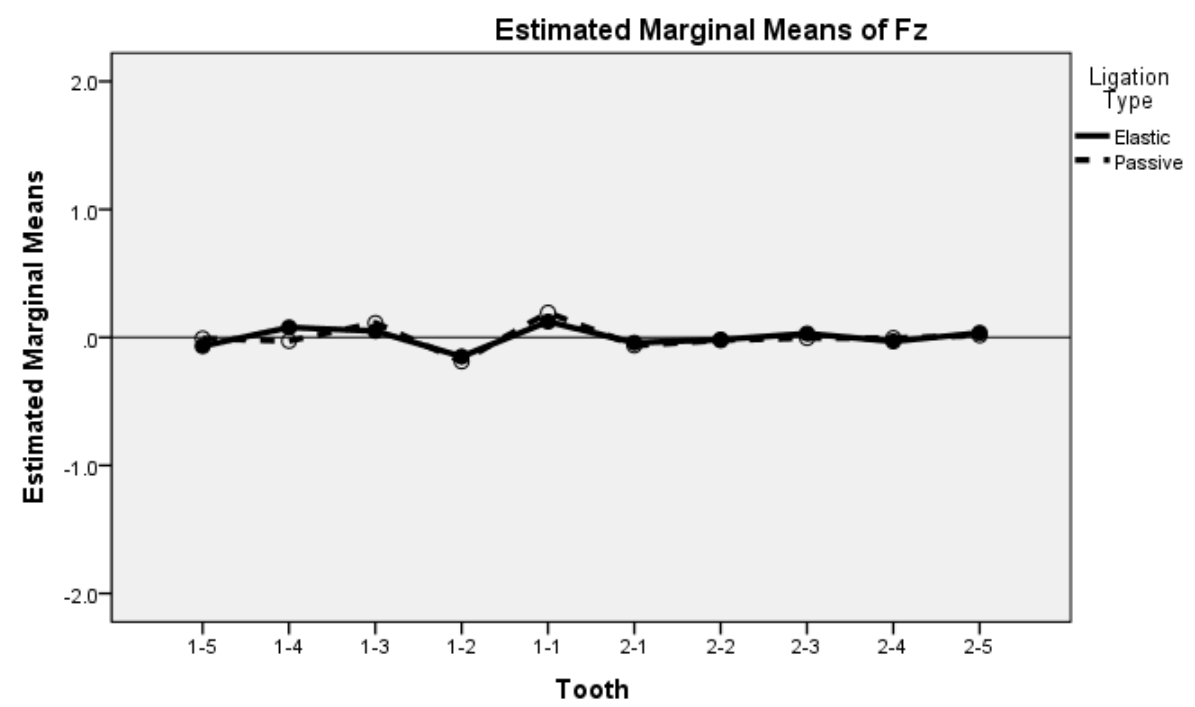

Fig. (4). Estimated marginal means of $F_{Z}$.

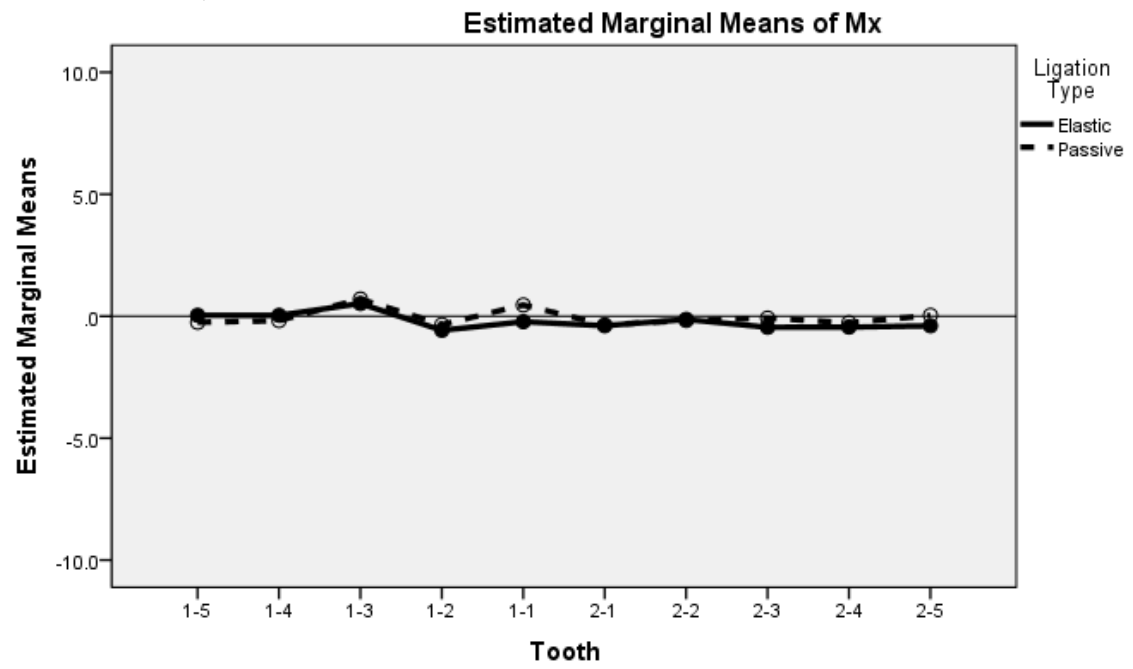

Fig. (5). Estimated marginal means of $M_{X}$.

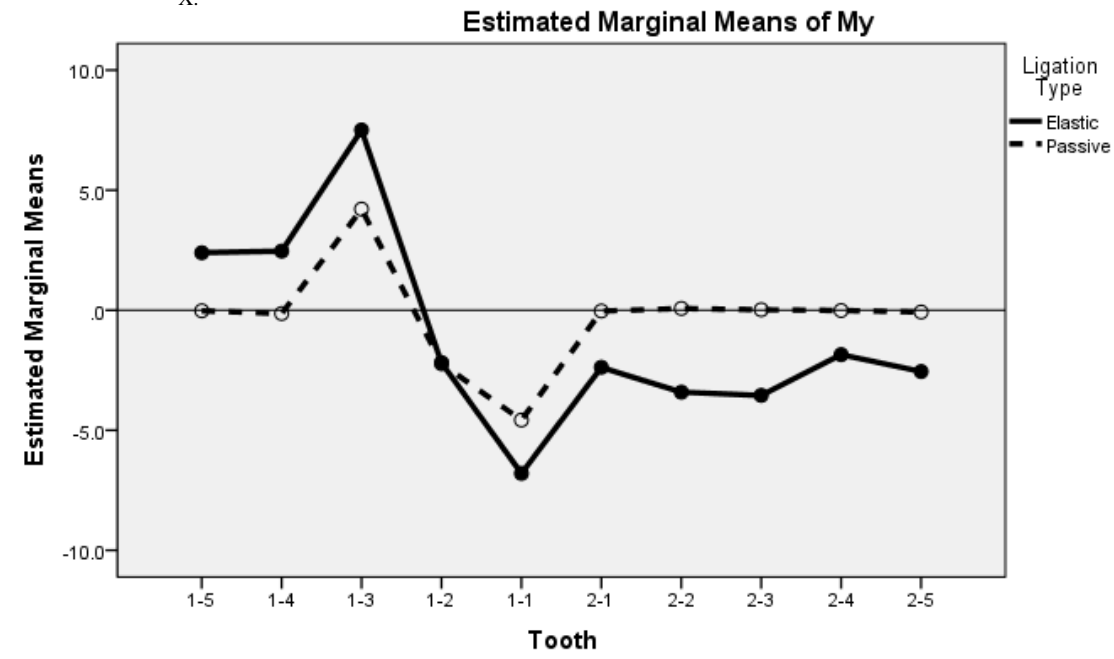

Fig. (6). Estimated marginal means of $\mathrm{M}_{\mathrm{Y}}$.

\section{DISCUSSION}

The objectives of this experiment were to determine whether elastic and passive ligation produce the same magnitude of forces and moments in a lingual lateral incisor experiment, and whether the forces and moments produced propagate along the arch equally.

Overall, these results show convincing evidence that the maximum forces and moments produced by elastic ligation 


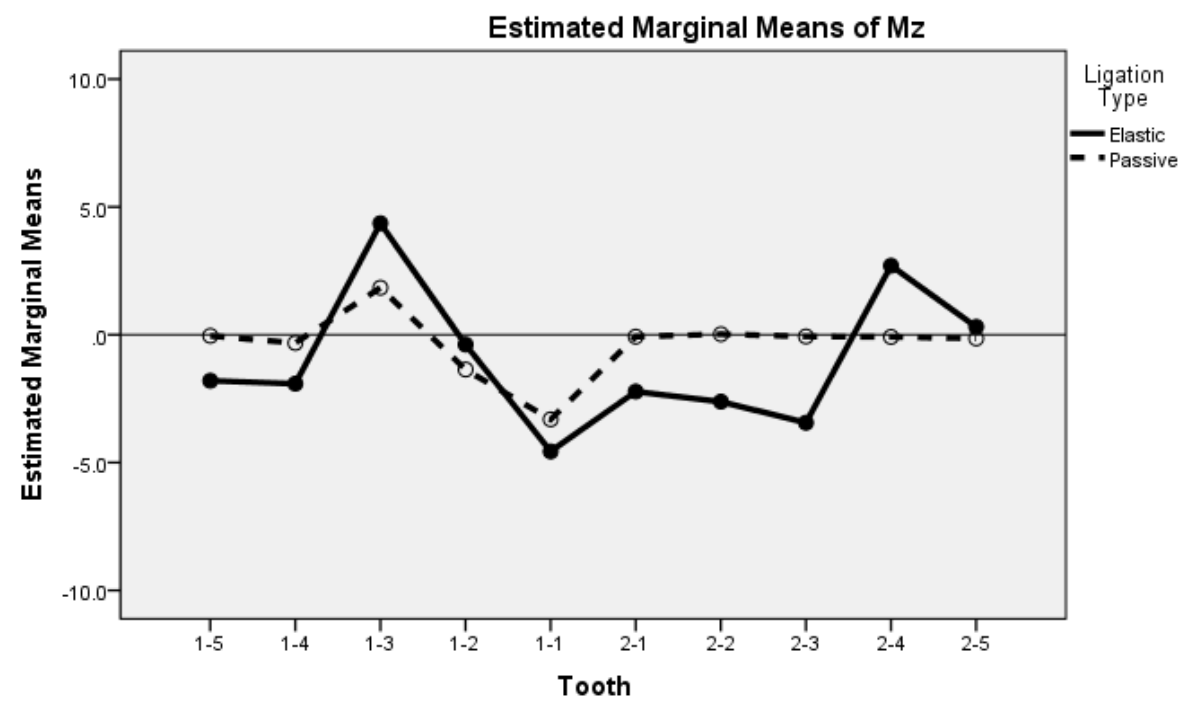

Fig. (7). Estimated marginal means of $\mathrm{M}_{\mathrm{Z}}$.

are not equal to those produced by passive ligation. The results suggest that the maximum forces and moments were consistently higher for elastic ligation than passive ligation for almost all outcome variables when all the teeth were considered jointly. The interaction factor between tooth position and ligation type illustrated convincing evidence that there is a difference in maximum force and moment values for all outcome variables between ligation types considering all tooth positions along the arch; this means that the propagation of forces and moments along the arch are different between the ligation types.

Forces along the $\mathrm{x}$-axis (the mesiodistal axis) in Fig. (2) represent resistance to sliding of the wire through the bracket. $F_{X}$ values are largest on the adjacent teeth (1-3 and 1-1), with higher values for elastic ligation than passive. In both ligations methods, $\mathrm{F}_{\mathrm{X}}$ has surpassed the $0.2 \mathrm{~N}$ threshold on the 1-1 and 1-3 teeth. Conversely, $F_{X}$ values on teeth beyond the 1-1 and 1-3 decay below clinically significant levels for passive ligation while remaining at, or near, this level for elastic ligation. This illustrates greater propagation of relevant sliding forces in elastic ligation compared to passive.

The wire responds to the lingual movement of 1-2 by flexing toward the front or back of the slot, which is represented by the y-axis (buccolingual) forces in Fig. (3). $\mathrm{F}_{\mathrm{Y}}$ values are largest on 1-2 and decrease as they propagate along the arch, but alternate between the positive (buccal) and negative (lingual) directions. This is more apparent in the elastic ligation trials; a potential reason for this might be because passively ligated brackets allow the wire to release its flexure, whereas elastically ligated wires prevent this release due to their active grip on the wire. Inspecting the propagation of $\mathrm{F}_{\mathrm{Y}}$ around the arch, it is again apparent that elastic ligation facilitates force magnitudes above $0.2 \mathrm{~N}$ well beyond 1-3 and 1-1. In contrast, passive ligation leads to decay of forces in adjacent teeth below clinically relevant levels.

For this experiment, the $\mathrm{F}_{\mathrm{Z}}$ forces (the occlusogingival axis) should be close to zero since the neither the tooth nor the wire was moved in the $\mathrm{z}$ direction. As can be seen in
Fig. (4), although the $\mathrm{F}_{\mathrm{Z}}$ forces do cross the threshold of 0.09 $\mathrm{N}$, they are much smaller in magnitude compared to $\mathrm{F}_{\mathrm{X}}$ and $F_{Y}$ and remain below the clinically relevant force level. As such, they can be considered irrelevant in discussing the mechanics of lingual incisor correction.

As expected, $\mathrm{M}_{\mathrm{X}}$ values are clinically irrelevant as well since $\mathrm{M}_{\mathrm{X}}$ represents torque and round wires do not allow for engagement between the wire and slot; this is presented in Fig. (5). Illustrated in Fig. (6), $\mathrm{M}_{Y}$, representing tipping moment, largely follows the same trend as $F_{X}$, both in terms of relative values and propagation along the arch between the two ligation types. $\mathrm{M}_{\mathrm{Z}}$, presented in Fig. (7), is the rotational moment of the teeth. The rotational moment of the neighboring teeth (1-3 and 1-1) about their long axes is due to the wire pushing along the slot $\left(F_{X}\right)$ while 1-2 is brought back to neutral position. Though $\mathrm{M}_{\mathrm{X}}$ and $\mathrm{M}_{\mathrm{Y}}$ values for the 1-1 and 1-3 using elastic ligation are in the range of clinically relevant moments, they are at the lower extreme which suggests they would have minimal, if any, effect on tooth movement. In all cases, moments for passively ligated brackets were below the $5.0 \mathrm{Nmm}$ threshold.

In general, it is apparent that clinically relevant force propagation around the arch for a lingual incisor was much more prevalent in elastic ligation compared to passive. Even though the force magnitude was close to the $0.2 \mathrm{~N}$ threshold in most cases, especially at the outer extremes from the 1-2 tooth, this is a consequence that should be avoided. Unwanted force propagation can lead to adverse effects which require further correction; eliminating such forces can lead to shortened treatment times and improved, more predictable, results.

Since the wires, brackets, and elastics were not randomly sampled from the wide selection of orthodontic manufacturers and material types, no inference to the general population can be made with the results of this experiment. However, inference to the population of these specific wires, brackets, and elastics can be made. Causal inference of ligation type can be made because the experiment trials were randomized between elastic and passive ligation types. Since only one malalignment was studied, the results and 
conclusions from this experiment cannot be generalized to all scenarios. Further investigation of different orthodontic malalignments and malocclusions is needed before generalizations can be made.

The OSIM allows users to determine forces and moments produced along the entire archform while moving one or more teeth in the occlusogingival or buccolingual directions. A unique strength to the OSIM and this study is the ability to demonstrate the propagation of forces and moments along the arch. However, several assumptions and simplifications were made for this study that may have affected the resultant force/moment system. These include the lack of tooth-totooth contact, soft tissue compliance, moisture, and intraoral disturbances such as mastication, lip pressure, and tongue pressure. These factors may affect resistance to sliding by allowing the release of binding between the wire and bracket, which may not occur in this study due to the rigidity of the current OSIM setup.

The time frame of this study is another factor that may have affected results. The tests were performed in short periods of time (a span of minutes) with new elastics for each test. This, along with the dry environment (lack of saliva), removes any effect of elastomeric aging present in the oral environment. A final limitation to this study was that passive brackets with their doors open were used to simulate conventional brackets in the elastic ligation trials. This was to ensure that the bracket and slot geometry were consistent between the ligation types, isolating the effect of the elastic as the only independent variable. It is possible that the combination of conventional brackets with elastic ligatures produces different force/moment systems due to differences in bracket geometry and the interface between the wire and slot.

While these factors must be considered before applying the results of this study in a clinical environment, this experiment does provide valuable insight as to how elastic and passive ligations behave differently in a lingual incisor malalignment. Strictly clinical studies are unable to measure loads produced by a given orthodontic appliance or malocclusion throughout the duration of treatment. As a result, overall conclusions regarding the treatment results can be drawn, yet discussion of intermediate treatment phases and appliance mechanics remain elusive. Furthermore, OSIM is currently the only in-vitro apparatus able to measure load propagation to each tooth around the arch. Use of this apparatus in scientific orthodontic research is vital to understanding appliance mechanics, and can be used in conjunction with clinical studies to interpret patient response. In this specific study, it was found that force and moment propagation around the arch was influenced by ligation method for a lingual incisor.

\section{CONCLUSION}

This study aimed to analyze the effects of elastic versus passive ligation on the magnitude and propagation of forces and moments produced along a continuous archform for a lingual lateral incisor malalignment. The hypotheses were that elastic ligation will produce greater magnitudes and propagate further than passive ligation. Both research questions for this experiment were answered qualitatively and quantitatively. The results of this experiment indicate that maximum forces and moments produced by elastic ligation are greater than those produced by passive ligation and that the magnitude of this difference for the mesiodistal $\left(F_{X}\right)$ and buccolingual $\left(F_{Y}\right)$ forces is clinically relevant. Additionally, it was determined that elastic ligation causes forces and moments to propagate further along the arch than passive ligation for all outcome variables.

\section{CONFLICT OF INTEREST}

The authors declare that there is no conflict of interest regarding the publication of this article.

\section{ACKNOWLEDGEMENTS}

Declared none.

\section{REFERENCES}

[1] J. Fok, R. W. Toogood, H. Badawi, J. P. Carey, and P. W. Major, "Analysis of maxillary arch force/couple systems for a simulated high canine malocclusion: Part 2. Elastic ligation," Angle Orthod., vol. 81, pp. 960-965, Nov. 2011.

[2] S. Kahlon, D. Rinchuse, J. M. Robison, and J. M. Close, "In-vitro evaluation of frictional resistance with 5 ligation methods and Gianelly-type working wires," Am. J. Orthod. Dentofacial Orthop., vol. 138 , pp. 67-71, July 2010 .

[3] A. P. T. Sims, N. E. Waters, D. J. Birnie, and R. J. Pethybridge, "A comparison of the forces required to produce tooth movement in vitro using two self-ligating brackets and a pre-adjusted bracket employing two types of ligation," Euro. J. Orthod., vol. 15, pp. 377-385, Oct. 1993.

[4] S. Thomas, M. Sherriff, and D. Birnie, "A comparative in vitro study of the frictional characteristics of two types of self-ligating brackets and two types of pre-adjusted edgewise brackets tied with elastomeric ligatures," Euro. J. Orthod., vol. 20, pp. 589-596, Oct. 1998.

[5] G. A. Thorstenson, and R. P. Kusy, "Resistance to sliding of selfligating brackets versus conventional stainless steel twin brackets with second-order angulation in the dry and wet (saliva) states," Am. J. Orthod. Dentofacial Orthop., vol. 120, pp. 361-370, Oct. 2001.

[6] N. W. T. Harradine, "Self-ligating brackets: where are we now?", $J$. Orthod., vol. 30, pp. 262-273, Sep. 2003.

[7] C. J. Burstone, and M. R. Marcotte, Problem Solving in Orthodontics: Goal-Oriented Treatment Strategies. Chicago: Quintessence Publishing, 2000.

[8] H. M. Badawi, R. W. Toogood, J. P. R. Carey, G. Heo, and P. W. Major, "Three-dimensional orthodontic force measurements," Am. J. Orthod. Dentofacial Orthop., vol. 136, pp. 518-528, Oct. 2009.

[9] H. M. Badawi, "The use of multi-axis force transducers for orthodontic foces and moments identification," $\mathrm{PhD}$ Thesis, University of Alberta, Edmonton, AB, Canada, 2009.

[10] R. W. Toogood, H. M. Badawi, J. Carey, A. Farys, I. Malis, E. Chen, L. Brenet, and P. Major, "Design of the orthodontic simulator (OSIM)", in Proceedings of the Canadian Society of Mechanical Engineering Forum, 2010.

[11] J. Fok, R. W. Toogood, H. Badawi, J. P. Carey, and P. W. Major, "Analysis of maxillary arch force/couple systems for a simulated high canine malocclusion: Part 1. Passive ligation," Angle Orthod., vol. 81, pp. 953-959, Nov. 2011.

[12] R. Nanda, Biomechanics in Clinical Orthodontics. Philadelphia: WB Saunders Co, 1996. 
[13] H. Gmyrek, C. Bourauel, G. Richter, and W. Harzer, "Torque capacity of metal and plastic brackets with reference to materials, application, technology, and biomechanics", J. Orofac. Orthop., vol. 63 , pp. 113-128, March 2002.

Received: July 24, 2014

Revised: September 09, 2014

Accepted: September 12, 2014

(C) Seru et al.; Licensee Bentham Open.

This is an open access article licensed under the terms of the Creative Commons Attribution Non-Commercial License (http://creativecommons.org/licenses/by-nc/3.0/) which permits unrestricted, non-commercial use, distribution and reproduction in any medium, provided the work is properly cited. 